\title{
The Value Distribution of Some Differential Polynomials
}

\author{
${ }^{1}$ Subhas S. Bhoosnurmath, ${ }^{2}$ K.S.L.N.Prasad \\ ${ }^{I}$ Department of Mathematics, Karnatak University, Dharwad-580003-INDIA \\ ${ }^{2}$ Associate Professor, Department of Mathematics, Karnatak Arts College, Dharwad-580001, INDIA
}

Abstract: We prove a value distribution theorem for meromorphic functions having few poles from which we obtain several interesting results which improve some results of W. Doeringer, C.C.Yang, A.P.Singh, G.P.Barker and others.

\section{Introduction And Main Results}

Let $\mathrm{f}$ be a transcendental meromorphic function in the plane.

A monomial in $\mathrm{f}$, is an expression of the form $\mathrm{M}[\mathrm{f}]=\mathrm{f}^{\mathrm{n}_{\mathrm{o}}}\left(\mathrm{f}^{(1)}\right)^{\mathrm{n}_{1}} \ldots\left(\mathrm{f}^{(\mathrm{k})}\right)^{\mathrm{n}_{\mathrm{k}}}$ where $\mathrm{n}_{0}, \mathrm{n}_{1}, \mathrm{n}_{2, \ldots}, \mathrm{n}_{\mathrm{k}}$ are non negative integers.

$$
\begin{aligned}
& \gamma_{\mathrm{M}}=\mathrm{n}_{0}+\mathrm{n}_{1}+\mathrm{n}_{2}+\ldots+\mathrm{n}_{\mathrm{k}} \quad \text { is called the degree of the monomial and } \\
& \Gamma_{\mathrm{M}}=\mathrm{n}_{0}+2 \mathrm{n}_{1}+\ldots+(\mathrm{k}+1) \mathrm{n}_{\mathrm{k}} \text {, the weight. }
\end{aligned}
$$

If $\mathrm{M}_{1}[\mathrm{f}], \mathrm{M}_{2}[\mathrm{f}], \ldots, \mathrm{M}_{\mathrm{n}}[\mathrm{f}]$ denote monomials in $\mathrm{f}$, then,

$\mathrm{Q}[\mathrm{f}]=\mathrm{a}_{1} \mathrm{M}_{1}[\mathrm{f}]+\mathrm{a}_{2} \mathrm{M}_{2}[\mathrm{f}]+\ldots+\mathrm{a}_{\mathrm{n}} \mathrm{M}_{\mathrm{n}}[\mathrm{f}]$, where $\mathrm{a}_{\mathrm{i}} \neq 0(\mathrm{i}=1,2, \ldots, \mathrm{n})$, is called a differential polynomial in $\mathbf{f}$ of degree $\gamma_{\mathrm{Q}}=\operatorname{Max}\left\{\gamma_{\mathrm{M}_{\mathrm{j}}}: 1 \leq \mathrm{j} \leq \mathrm{n}\right\}$ and weight $\quad \Gamma_{Q}=\operatorname{Max}\left\{\Gamma_{M_{j}}: 1 \leq j \leq n\right\}$

Also, we call the numbers $\underline{\gamma_{Q}}=\min _{1 \leq \mathrm{j} \leq \mathrm{n}} \gamma_{\mathrm{M}_{\mathrm{j}}}$ and $\mathrm{k}$ (the order of the highest derivative of $\mathrm{f}$ ) the lower degree and the order of $\mathrm{Q}[\mathrm{f}]$ respectively. If $\gamma_{\mathrm{Q}}=\gamma_{\mathrm{Q}}, \mathrm{Q}[\mathrm{f}]$ is called a homogeneous differential

\section{II. polynomial.}

W.K.Hayman in his well known problem book 'Problems in Function Theory' has raised some interesting open problems related to the value distribution of differential polynomials.

In 1988, Hong-XunYi[6] proved the following result:

Theorem A: Let $\mathrm{f}$ be transcendental meromorphic function in the plane and $\mathrm{Q}_{1}[\mathrm{f}] \not \equiv 0, \mathrm{Q}_{2}[\mathrm{f}] \not \equiv 0$ be differential polynomials in $\mathrm{f}$.

Let $P_{1}[f]=a_{n} f^{n}+a_{n-1} f^{n-1}+\ldots \ldots \ldots+a_{0}\left(a_{n}(z) \not \equiv 0\right)$

If $\mathrm{F}=\mathrm{P}_{1}[\mathrm{f}] \mathrm{Q}_{1}[\mathrm{f}]+\mathrm{Q}_{2}[\mathrm{f}]$, then

$$
\left(\mathrm{n}-\gamma_{\mathrm{Q}_{2}}\right) \mathrm{T}(\mathrm{r}, \mathrm{f}) \leq \overline{\mathrm{N}}\left(\mathrm{r}, \frac{1}{\mathrm{~F}}\right)+\overline{\mathrm{N}}\left(\mathrm{r}, \frac{1}{\mathrm{P}_{1}[\mathrm{f}]}\right)+\left(\Gamma_{\mathrm{Q}_{2}}-\gamma_{\mathrm{Q}_{2}}+1\right) \overline{\mathrm{N}}(\mathrm{r}, \mathrm{f})+\mathrm{S}(\mathrm{r}, \mathrm{f}) \text {. }
$$

Thinking on the same lines, we considered a different combination of differential polynomials and obtained an interesting result which generalizes the result of Hong-Xun Yi.

The following theorem is our main result.

Theorem 1: Let $\mathrm{f}$ be transcendental meromorphic function in the plane and

$\mathrm{Q}_{1}[\mathrm{f}] \not \equiv 0, \mathrm{Q}_{2}[\mathrm{f}] \not \equiv 0$ be differential polynomials in $\mathrm{f}$.

$$
\begin{aligned}
& \text { Let } P_{1}[f]=a_{n} f^{n}+a_{n-1} f^{n-1}+\ldots \ldots \ldots+a_{0} \quad\left(a_{n}(z) \neq \equiv\right) \\
& \text { and } P_{2}[f]=b_{m} f^{m}+b_{m-1} f^{m-1}+\ldots \ldots \ldots+b_{0}\left(b_{m}(z) \neq 0\right) \\
& \text { where } n>m . \\
& \text { If } F=P_{1}[f] Q_{1}[f]+P_{2}[f] Q_{2}[f]
\end{aligned}
$$


Then, $\left(\mathrm{n}-\gamma_{\mathrm{Q}_{2}}\right) \mathrm{T}(\mathrm{r}, \mathrm{f}) \leq \overline{\mathrm{N}}\left(\mathrm{r}, \frac{1}{\mathrm{~F}}\right)+\overline{\mathrm{N}}\left(\mathrm{r}, \frac{1}{\mathrm{P}_{1}[\mathrm{f}]}\right)+3 \overline{\mathrm{N}}\left(\mathrm{r}, \frac{1}{\mathrm{P}_{2}[\mathrm{f}]}\right)+\left(\Gamma_{\mathrm{Q}_{2}}-\gamma_{\mathrm{Q}_{2}}+1\right) \overline{\mathrm{N}}(\mathrm{r}, \mathrm{f})+\mathrm{S}(\mathrm{r}, \mathrm{f})$.

To prove the above Theorem, we require the following Lemmas.

Lemma 1[2]: Suppose $P_{1}[f]$ is as in (1).

Then, $\mathrm{m}\left(\mathrm{r}, \mathrm{P}_{1}[\mathrm{f}]\right)=\mathrm{n} \mathrm{m}(\mathrm{r}, \mathrm{f})+\mathrm{S}(\mathrm{r}, \mathrm{f})$

Lemma 2 [4]: Let $f(z)$ be a transcendental meromorphic function, $P[f]$ and $Q[f]$ be differential polynomials in $f$. If degree of $Q[f]$ is at most $n$ and $f^{n} P[f]=Q[f]$, then, $\quad m(r, P[f])=S(r, f)$.

Lemma 3 [3]: If $P[f]$ is a homogeneous differential polynomial of degree $n$, then,

$$
\mathrm{m}\left(\mathrm{r}, \frac{\mathrm{P}[\mathrm{f}]}{\mathrm{f}^{\mathrm{n}}}\right)=\mathrm{S}(\mathrm{r}, \mathrm{f}) \text {. }
$$

Lemma 4 [6]: Suppose that $Q[f]$ is a differential polynomial in $f$. Let $z_{0}$ be a pole of $f$ of order $m$ and not a zero or a pole of the co-efficients of $Q[f]$. Then $z_{0}$ is a pole of $Q[f]$ of order atmost $m \gamma_{Q}+\left(\Gamma_{Q}-\gamma_{Q}\right)$.

Lemma 5 [11]: If $Q[f]$ is a differential polynomial in $f$ with arbitrary meromorphic co-efficients $q_{j}$, then

$$
\mathrm{m}(\mathrm{r}, \mathrm{Q}[\mathrm{f}]) \leq \gamma_{\mathrm{Q}} \mathrm{m}(\mathrm{r}, \mathrm{f})+\sum_{\mathrm{j}=1}^{\mathrm{n}} \mathrm{m}\left(\mathrm{r}, \mathrm{q}_{\mathrm{j}}\right)+\mathrm{S}(\mathrm{r}, \mathrm{f}) .
$$

\section{Proof of Theorem 1:}

We have $F=P_{1}[\mathrm{f}] Q_{1}[\mathrm{f}]+P_{2}[\mathrm{f}] Q_{2}[\mathrm{f}]$

Therefore, $F^{\prime}=\frac{F^{\prime}}{F} P_{1}[f] Q_{1}[f]+\frac{F^{\prime}}{F} P_{2}[f] Q_{2}[f]$

$$
\text { Also, } F^{\prime}=P_{1}[\mathrm{f}]\left(\mathrm{Q}_{1}[\mathrm{f}]\right)^{\prime}+\mathrm{Q}_{1}[\mathrm{f}]\left(\mathrm{P}_{1}[\mathrm{f}]\right)^{\prime}+\mathrm{P}_{2}[\mathrm{f}]\left(\mathrm{Q}_{2}[\mathrm{f}]\right)^{\prime}+\mathrm{Q}_{2}[\mathrm{f}]\left(\mathrm{P}_{2}[\mathrm{f}]\right)^{\prime}
$$

Hence, we have

$$
\begin{aligned}
\frac{F^{\prime}}{F} P_{1}[f] Q_{1}[f]+\frac{F^{\prime}}{F} P_{2}[f] Q_{2}[f] & =P_{1}[f]\left(Q_{1}[f]\right)^{\prime}+Q_{1}[f]\left(P_{1}[f]\right)^{\prime} \\
& +P_{2}[f]\left(Q_{2}[f]\right)^{\prime}+Q_{2}[f]\left(P_{2}[f]\right)^{\prime}
\end{aligned}
$$

Therefore,

$$
\begin{aligned}
& \mathrm{P}_{1}[\mathrm{f}]\left[\frac{\mathrm{F}^{\prime}}{\mathrm{F}} \mathrm{Q}_{1}[\mathrm{f}]-\left(\mathrm{Q}_{1}[\mathrm{f}]\right)^{\prime}-\frac{\left(\mathrm{P}_{1}[\mathrm{f}]\right)^{\prime} \mathrm{Q}_{1}[\mathrm{f}]}{\mathrm{P}_{1}[\mathrm{f}]}\right]=\mathrm{P}_{2}[\mathrm{f}]\left[\left(\mathrm{Q}_{2}[\mathrm{f}]\right)^{\prime}-\frac{\mathrm{F}^{\prime}}{\mathrm{F}} \mathrm{Q}_{2}[\mathrm{f}]+\frac{\left(\mathrm{P}_{2}[\mathrm{f}]\right)^{\prime} \mathrm{Q}_{2}[\mathrm{f}]}{\mathrm{P}_{2}[\mathrm{f}]}\right] . \\
& \text { Or, } \mathrm{P}_{1}[\mathrm{f}] .\left\{\frac{1}{\mathrm{P}_{2}[\mathrm{f}]}\left[\frac{\mathrm{F}^{\prime}}{\mathrm{F}} \mathrm{Q}_{1}[\mathrm{f}]-\left(\mathrm{Q}_{1}[\mathrm{f}]\right)^{\prime}-\frac{\left(\mathrm{P}_{1}[\mathrm{f}]\right)^{\prime} \mathrm{Q}_{1}[\mathrm{f}]}{\mathrm{P}_{1}[\mathrm{f}]}\right]\right\}=\left[\left(\mathrm{Q}_{2}[\mathrm{f}]\right)^{\prime}-\frac{\mathrm{F}^{\prime}}{\mathrm{F}} \mathrm{Q}_{2}[\mathrm{f}]+\frac{\left(\mathrm{P}_{2}[\mathrm{f}]\right)^{\prime} \mathrm{Q}_{2}[\mathrm{f}]}{\mathrm{P}_{2}[\mathrm{f}]}\right],
\end{aligned}
$$

which is of the form, $P_{1}[f] Q *[f]=Q[f]$,

$$
\begin{aligned}
\text { where, } Q^{*}[\mathrm{f}]=\frac{F^{\prime}}{F_{2}[f]} Q_{1}[f]-\frac{\left(Q_{1}[f]\right)^{\prime}}{P_{2}[f]}-\frac{\left(P_{1}[f]\right)^{\prime} Q_{1}[f]}{P_{1}[f] P_{2}[f]} \\
\text { and } Q[f]=\left(Q_{2}[f]\right)^{\prime}-\frac{F^{\prime}}{F} Q_{2}[f]+\frac{\left(P_{2}[f]\right)^{\prime} Q_{2}[f]}{P_{2}[f]}
\end{aligned}
$$

Without loss of generality, let us assume that $\mathrm{Q}[\mathrm{f}] \not \equiv 0$.

By Lemma $2, \mathrm{~m}(\mathrm{r}, \mathrm{Q} *[\mathrm{f}])=\mathrm{S}(\mathrm{r}, \mathrm{f})$

Again from (4), $\mathrm{P}_{1}[\mathrm{f}]=\frac{\mathrm{Q}[\mathrm{f}]}{\mathrm{Q} *[\mathrm{f}]}$ 
Therefore, $\quad \mathrm{m}\left(\mathrm{r}, \mathrm{P}_{1}[\mathrm{f}]\right) \leq \mathrm{m}(\mathrm{r}, \mathrm{Q}[\mathrm{f}])+\mathrm{m}\left(\mathrm{r}, \frac{1}{\mathrm{Q} *[\mathrm{f}]}\right)$

Again by Lemma 5 and (5), $m(r, Q[f]) \leq \gamma_{\mathrm{Q}_{2}} \mathrm{~m}(\mathrm{r}, \mathrm{f})+\mathrm{S}(\mathrm{r}, \mathrm{f})$

From the First Fundamental Theorem, we have

$$
\mathrm{m}\left(\mathrm{r}, \frac{1}{\mathrm{Q}^{*}[\mathrm{f}]}\right)=\mathrm{N}\left(\mathrm{r}, \mathrm{Q}^{*}[\mathrm{f}]\right)-\mathrm{N}\left(\mathrm{r}, \frac{1}{\mathrm{Q}^{*}[\mathrm{f}]}\right)+\mathrm{S}(\mathrm{r}, \mathrm{f})
$$

Also, all the poles of $Q *[f]$ occur at the zeros of $F, P_{1}[f]$ and $P_{2}[f]$, the pole of $f$, and the zeros and poles of the co-efficients. Suppose $z_{0}$ is a pole of $f$ of order $m$. Then $z_{0}$ is a pole $P_{1}[f]$ of order $m n$.

From Lemma $4, \mathrm{z}_{0}$ is a pole of $\mathrm{Q}[\mathrm{f}]$ of order atmost $\mathrm{m} \gamma_{\mathrm{Q}_{2}}+\left(\Gamma_{\mathrm{Q}_{2}}-\gamma_{\mathrm{Q}_{2}}+1\right)$

If $\mathrm{z}_{0}$ is a pole of $\mathrm{Q} *[\mathrm{f}]$, since $\mathrm{Q} *[\mathrm{f}]=\frac{\mathrm{Q}[\mathrm{f}]}{\mathrm{P}_{1}[\mathrm{f}]}$,

$\mathrm{z}_{0}$ is a pole of $\mathrm{Q}^{*}[\mathrm{f}]$ of order atmost $\mathrm{m} \gamma_{\mathrm{Q}_{2}}+\left(\Gamma_{\mathrm{Q}_{2}}-\gamma_{\mathrm{Q}_{2}}+1\right)-\mathrm{mn}$

$$
=\left(\Gamma_{\mathrm{Q}_{2}}-\gamma_{\mathrm{Q}_{2}}+1\right)-\mathrm{m}\left(\mathrm{n}-\gamma_{\mathrm{Q}_{2}}\right)
$$

If $\mathrm{z}_{0}$ is a pole of $\mathrm{Q} *[\mathrm{f}]$ then since $\frac{1}{\mathrm{Q} *[\mathrm{f}]}=\frac{\mathrm{P}_{1}[\mathrm{f}]}{\mathrm{Q}[\mathrm{f}]}$,

$\mathrm{z}_{0}$ is a zero of $\frac{1}{\mathrm{Q}^{*}[\mathrm{f}]}$ of order atleast $\mathrm{mn}-\left\{\mathrm{m} \gamma_{\mathrm{Q}_{2}}+\left(\Gamma_{\mathrm{Q}_{2}}-\gamma_{\mathrm{Q}_{2}}+1\right)\right\}$

$$
=m\left(n-\gamma_{Q_{2}}\right)-\left(\Gamma_{Q_{2}}-\gamma_{Q_{2}}+1\right)
$$

Thus, we have,

$$
\begin{aligned}
& \mathrm{N}(\mathrm{r}, \mathrm{Q} *[\mathrm{f}])-\mathrm{N}\left(\mathrm{r}, \frac{1}{\mathrm{Q} *[\mathrm{f}]}\right) \leq \overline{\mathrm{N}}\left(\mathrm{r}, \frac{1}{\mathrm{~F}}\right)+\overline{\mathrm{N}}\left(\mathrm{r}, \frac{1}{\mathrm{P}_{1}[\mathrm{f}]}\right)+3 \overline{\mathrm{N}}\left(\mathrm{r}, \frac{1}{\mathrm{P}_{2}[\mathrm{f}]}\right) \\
&+\left(\Gamma_{\mathrm{Q}_{2}}-\gamma_{\mathrm{Q}_{2}}+1\right) \overline{\mathrm{N}}(\mathrm{r}, \mathrm{f})-\left(\mathrm{n}-\gamma_{\mathrm{Q}_{2}}\right) \mathrm{N}(\mathrm{r}, \mathrm{f})+\mathrm{S}(\mathrm{r}, \mathrm{f})
\end{aligned}
$$

In view of (7), (8), (9), the equation (6) becomes,

$$
\begin{aligned}
\mathrm{nm}(\mathrm{r}, \mathrm{f}) & \leq \gamma_{\mathrm{Q}_{2}} \mathrm{~m}(\mathrm{r}, \mathrm{f})+\overline{\mathrm{N}}\left(\mathrm{r}, \frac{1}{\mathrm{~F}}\right)+\overline{\mathrm{N}}\left(\mathrm{r}, \frac{1}{\mathrm{P}_{1}[\mathrm{f}]}\right)+3 \overline{\mathrm{N}}\left(\mathrm{r}, \frac{1}{\mathrm{P}_{2}[\mathrm{f}]}\right) \\
& +\left(\Gamma_{\mathrm{Q}_{2}}-\gamma_{\mathrm{Q}_{2}}+1\right) \overline{\mathrm{N}}(\mathrm{r}, \mathrm{f})-\left(\mathrm{n}-\gamma_{\mathrm{Q}_{2}}\right) \mathrm{N}(\mathrm{r}, \mathrm{f})+\mathrm{S}(\mathrm{r}, \mathrm{f}) .
\end{aligned}
$$

Hence, we have

$$
\begin{aligned}
\left(\mathrm{n}-\gamma_{\mathrm{Q}_{2}}\right) \mathrm{T}(\mathrm{r}, \mathrm{f}) & \leq \overline{\mathrm{N}}\left(\mathrm{r}, \frac{1}{\mathrm{~F}}\right)+\overline{\mathrm{N}}\left(\mathrm{r}, \frac{1}{\mathrm{P}_{1}[\mathrm{f}]}\right)+3 \overline{\mathrm{N}}\left(\mathrm{r}, \frac{1}{\mathrm{P}_{2}[\mathrm{f}]}\right) \\
& +\left(\Gamma_{\mathrm{Q}_{2}}-\gamma_{\mathrm{Q}_{2}}+1\right) \overline{\mathrm{N}}(\mathrm{r}, \mathrm{f})+\mathrm{S}(\mathrm{r}, \mathrm{f}) .
\end{aligned}
$$

Hence the result.

Putting $P_{1}[f]=f^{n}$ and $P_{2}[f]=f^{n-1}$ in the above theorem, we get the following.

Theorem 2: Let $\mathrm{f}, \mathrm{Q}_{1}[\mathrm{f}], \mathrm{Q}_{2}[\mathrm{f}]$ be as defined in Theorem 1 .

$$
\text { Let } F=f^{n} Q_{1}[f]+f^{n-1} Q_{2}[f] \text {. }
$$

Then,

$$
\left(\mathrm{n}-\gamma_{\mathrm{Q}_{2}}\right) \mathrm{T}(\mathrm{r}, \mathrm{f}) \leq \overline{\mathrm{N}}\left(\mathrm{r}, \frac{1}{\mathrm{~F}}\right)+4 \overline{\mathrm{N}}\left(\mathrm{r}, \frac{1}{\mathrm{f}}\right)+\left(\Gamma_{\mathrm{Q}_{2}}-\gamma_{\mathrm{Q}_{2}}+1\right) \overline{\mathrm{N}}(\mathrm{r}, \mathrm{f})+\mathrm{S}(\mathrm{r}, \mathrm{f})
$$

If $\mathrm{P}_{2}[\mathrm{f}] \equiv 1$, we get the following.

Theorem 3: Let $P_{1}[f], Q_{1}[f]$ and $Q_{2}[f]$ be as defined in Theorem 1 . 
If $\mathrm{F}=\mathrm{P}_{1}[\mathrm{f}] \mathrm{Q}_{1}[\mathrm{f}]+\mathrm{Q}_{2}[\mathrm{f}]$, then

$$
\left(\mathrm{n}-\gamma_{\mathrm{Q}_{2}}\right) \mathrm{T}(\mathrm{r}, \mathrm{f}) \leq \overline{\mathrm{N}}\left(\mathrm{r}, \frac{1}{\mathrm{~F}}\right)+\overline{\mathrm{N}}\left(\mathrm{r}, \frac{1}{\mathrm{P}_{1}[\mathrm{f}]}\right)+\left(\Gamma_{\mathrm{Q}_{2}}-\gamma_{\mathrm{Q}_{2}}+1\right) \overline{\mathrm{N}}(\mathrm{r}, \mathrm{f})+\mathrm{S}(\mathrm{r}, \mathrm{f})
$$

which is the result of Hong Xun Yi [5].

As an application of theorem 2, we have the following.

Theorem 4: Let $F=f^{n} \mathrm{Q}[\mathrm{f}]$ where $\mathrm{Q}[\mathrm{f}]$ is a differential polynomials in $\mathrm{f}$.

If $\mathrm{n} \geq 1$, then $\rho_{\mathrm{F}}=\rho_{\mathrm{f}}$ and $\lambda_{\mathrm{F}}=\lambda_{\mathrm{f}}$

Proof : If $F=f^{n} Q[f]$, then by theorem2, we have

$$
\mathrm{nT}(\mathrm{r}, \mathrm{f}) \leq \overline{\mathrm{N}}\left(\mathrm{r}, \frac{1}{\mathrm{~F}}\right)+4 \overline{\mathrm{N}}\left(\mathrm{r}, \frac{1}{\mathrm{f}}\right)+\overline{\mathrm{N}}(\mathrm{r}, \mathrm{f})+\mathrm{S}(\mathrm{r}, \mathrm{f}) .
$$

Clearly, the zeros and poles of $\mathrm{f}$ are that of $\mathrm{F}$ respectively.

Therefore, $4 \overline{\mathrm{N}}\left(\mathrm{r}, \frac{1}{\mathrm{f}}\right)+\overline{\mathrm{N}}(\mathrm{r}, \mathrm{f}) \leq 4 \overline{\mathrm{N}}\left(\mathrm{r}, \frac{1}{\mathrm{~F}}\right)+\overline{\mathrm{N}}(\mathrm{r}, \mathrm{F})+\mathrm{S}(\mathrm{r}, \mathrm{f})$

Therefore, $n \mathrm{~T}(\mathrm{r}, \mathrm{f}) \leq \overline{\mathrm{N}}\left(\mathrm{r}, \frac{1}{\mathrm{~F}}\right)+4 \overline{\mathrm{N}}\left(\mathrm{r}, \frac{1}{\mathrm{f}}\right)+\overline{\mathrm{N}}(\mathrm{r}, \mathrm{f})+\mathrm{S}(\mathrm{r}, \mathrm{f})$

$$
\leq 6 \mathrm{~T}(\mathrm{r}, \mathrm{F})+\mathrm{S}(\mathrm{r}, \mathrm{f})
$$

Therefore, $\mathrm{T}(\mathrm{r}, \mathrm{f})=\mathrm{O}\{\mathrm{T}(\mathrm{r}, \mathrm{F})\}$ as $\mathrm{r} \rightarrow \infty$.

Also, we know that $\mathrm{T}(\mathrm{r}, \mathrm{F})=\mathrm{O}\{\mathrm{T}(\mathrm{r}, \mathrm{f})\}$ as $\mathrm{r} \rightarrow \infty$.

Hence the Theorem.

Theorem 5: No transcendental meromorphic function $f$ with $\bar{N}(r, f)+\bar{N}\left(r, \frac{1}{f}\right)=S(r, f)$

can satisfy an equation of the form

$$
\mathrm{F}=\mathrm{a}_{1} \mathrm{f}^{\mathrm{n}} \mathrm{Q}_{1}[\mathrm{f}]+\mathrm{a}_{2} \mathrm{f}^{\mathrm{n}-1} \mathrm{Q}_{2}[\mathrm{f}]+\mathrm{a}_{3}=0
$$

where $a_{1} \not \equiv 0, a_{3} \not \equiv 0, n$ is a positive integer with $n>\gamma_{Q_{2}}, Q_{1}[f]$ and $Q_{2}[f]$ are differential polynomials in $\mathrm{f}$.

Proof: Suppose there exists a transcendental meromorphic function $\mathrm{f}$ satisfying (9).

Then by theorem 2, we have,

$$
\begin{aligned}
\left(\mathrm{n}-\gamma_{\mathrm{Q}_{2}}\right) \mathrm{T}(\mathrm{r}, \mathrm{f}) & \leq \overline{\mathrm{N}}\left(\mathrm{r}, \frac{1}{\mathrm{~F}}\right)+4 \overline{\mathrm{N}}\left(\mathrm{r}, \frac{1}{\mathrm{f}}\right)+\left(\Gamma_{\mathrm{Q}_{2}}-\gamma_{\mathrm{Q}_{2}}+1\right) \overline{\mathrm{N}}(\mathrm{r}, \mathrm{f})+\mathrm{S}(\mathrm{r}, \mathrm{f}) \\
& \leq 5 \overline{\mathrm{N}}\left(\mathrm{r}, \frac{1}{\mathrm{f}}\right)+\mathrm{S}(\mathrm{r}, \mathrm{f}) \\
\text { Or, } \mathrm{n}-\gamma_{\mathrm{Q}_{2}} & \leq \frac{5 \overline{\mathrm{N}}\left(\mathrm{r}, \frac{1}{\mathrm{f}}\right)+\mathrm{S}(\mathrm{r}, \mathrm{f})}{\mathrm{T}(\mathrm{r}, \mathrm{f})}=\frac{\mathrm{S}(\mathrm{r}, \mathrm{f})}{\mathrm{T}(\mathrm{r}, \mathrm{f})} \text { by hypothesis. }
\end{aligned}
$$

Or, $\mathrm{n}-\gamma_{\mathrm{Q}_{2}} \leq 0$, which implies $\mathrm{n} \leq \gamma_{\mathrm{Q}_{2}}$ which contradicts the choice of $\mathrm{n}$.

Hence the result.

This improves our earlier result namely

Theorem A [10]: No transcendental meromorphic function $\mathrm{f}$ with $\mathrm{N}(\mathrm{r}, \mathrm{f})=\mathrm{S}(\mathrm{r}, \mathrm{f})$ can satisfy the equation

$$
\mathrm{a}_{1}(\mathrm{z})[\mathrm{f}(\mathrm{z})]^{\mathrm{n}} \pi_{1}(\mathrm{f})+\mathrm{a}_{2}(\mathrm{z})[\mathrm{f}(\mathrm{z})]^{\mathrm{n}-1} \pi_{2}(\mathrm{f})+\mathrm{a}_{3}(\mathrm{z})=0,
$$

where $\pi_{1}(\mathrm{f})$ and $\pi_{2}(\mathrm{f})$ are differential polynomials of degree $n$ and $n-1$ respectively and $n>1$, and $\operatorname{Max}\left\{\operatorname{deg}\left(\mathrm{f}^{\mathrm{n}-1} \pi_{2}(\mathrm{f})\right)\right\}<$ n. and $\mathrm{a}_{1}(\mathrm{z}) \not \equiv 0$. 


\section{Acknowledgement:}

The second author is extremely thankful to University Grants Commission for the financial assistance given in the tenure of which this paper was prepared.

\section{References}

[1] BARKER G. P. and SINGH A. P. (1980): Commentarii mathematici universitatis : Sancti Pauli $29,183$.

[2] GUNTER FRANK and SIMON HELLERSTEIN (1986) : 'On the meromorphic solutions of non homogeneous linear differential equation with polynomial co-efficients', Proc. London Math. Soc. (3), 53, 407-428.

[3] HAYMAN W. K. (1964) : Meromorphic functions, Oxford Univ. Press, London.

[4] HONG-XUN YI (1990): ‘On a result of Singh', Bull. Austral. Math. Soc. Vol. 41 (1990) 417-420.

[5] HONG-XUN YI (1991): "On the value distribution of differential polynomials", Jl of Math. Analysis and applications 154, 318328.

[6] R. NEVANLINNA (1970) : Analytic functions, Springer-Verlag, New York.

[7] SINGH A. P. and DUKANE S. V. (1989): Some notes on differential polynomial proc. Nat. Acad. Sci. India 59 (A), II.

[8] SINGH A. P. and RAJSHREE DHAR (1993): Bull. Cal. Math. Soc. 85, 171-176.

[9] SUBHAS S. BHOOSNURMATH and CHHAYA M. HOMBALI (1998) : Fix points of certain differential polynomials, Proc. Ind. Acad. Sci. Vol. 108, No. 2, June-1998, pp. 121-131.

[10] YANG C. C. (1972) : 'A note on Malmquist's theorem on first order differential equations, ordering differential equations', Academic press, New York.

[11] YANG LO, (1982) : Value distribution theory, Science Press, Beijing,

[12] ZHAN-XIAOPING (1994): "Picard sets and value distributions for differential polynomials", J1. of Math. Ana. and Appl. 182, 722730 . 